\title{
Erythema nodosum induced by Covid-19 Pfizer-BioNTech mRNA Vaccine
}

\author{
FERDAOUS CHAHED ${ }^{1}$, Najah Ben Fadhel ${ }^{1}$, Haifa Ben Romdhane ${ }^{1}$, Monia Youssef ${ }^{1}$, \\ Amel Chaabane ${ }^{1}$, Karim Aouam ${ }^{1}$, and Nadia Ben fredj ${ }^{1}$ \\ ${ }^{1}$ Fattouma Bourguiba University Hospital of Monastir
}

January 12, 2022

\begin{abstract}
Erythema nodosum (EN), the most form of panniculitis, is mainly caused by numerous infective (especially Beta-hemolytic streptococcal infections) and non-infective (especially sarcoidosis) diseases and drugs. EN associated with vaccines has been rarely reported. We describe herein, an original clinical observation of EN induced by BNT162b2, an mRNA vaccine. A 75-year-old woman presented with diffuse erythematous painful rounded nodular lesions, located symmetrically over her legs. Six days before, she had received the second dose of Covid-19 vaccine (BNT162b2 (Pfizer-BioNTech)), followed by a sudden asthenia, polyarthralgia, throbbing and edema over her lower limbs. She had been given the first dose of the same Covid-19 vaccine 29 days prior to the second without incident. General physical examination was normal. Skin examination showed multiple, erythematous tender, nodules, $10-30 \mathrm{~mm}$ in diameter, over the tibial area. Complete blood count, renal and hepatic tests, antistreptolysin $\mathrm{O}$ titer, antinuclear antibody, thyroid test and chest radiograph and PCR, were carried out, and found to be normal. Histopathology revealed infiltration of deep dermal vessels and subcutaneous fat with lymphomononuclear cells and neutrophils, consistent with erythema nodosum. Treatment with analgesics led to complete resolution of the lesion after three months. The patient has shown no relapse after follow-up for three months. In conclusion, to our knowledge, this is the first case of EN induced by the second dose of BNT162b2 (Pfizer-BioNTech) Covid-19 vaccine. It is important for clinicians to be aware of this rare, yet potential, adverse effect to this vaccine.
\end{abstract}

Erythema nodosum induced by Covid-19 Pfizer-BioNTech mRNA Vaccine.

Ferdaous Chahed ${ }^{1, *}$, Najah Ben Fadhel ${ }^{1}$, Haifa Ben Romdhane ${ }^{1}$, Monia Youssef ${ }^{2}$, Amel Chaabane ${ }^{1}$, Karim Aouam ${ }^{1}$, Nadia Ben Fredj ${ }^{1}$

1: Department of Clinical Pharmacology. University Hospital/ Faculty of Medicine of Monastir. University of Monastir. Tunisia

2: Department of Dermatology. University Hospital of Monastir. University of Monastir. Tunisia

Corresponding author : Dr Ferdaous Chahed

Department of Clinical Pharmacology. University Hospital/ Faculty of Medicine of Monastir. University of Monastir. Tunisia

Tel :+21692 232122

e-mail :dr.ferdaws.chahed@gmail.com

Financial relationship with a biotechnology and/or pharmaceutical manufacturer:

NONE

Word count: 996 
The patient has read this draft (including the picture) and has given us consent to submit for publication.

Abstract : Erythema nodosum (EN), the most form of panniculitis, is mainly caused by numerous infective (especially Beta-hemolytic streptococcal infections), autoimmune diseases (especially sarcoidosis) and drugs. EN associated with vaccines has been rarely reported. We describe herein, an original clinical observation of EN induced by BNT162b2, an mRNA vaccine.

A 75-year-old woman presented with diffuse erythematous painful rounded nodular lesions, located symmetrically over her legs. Six days before, she had received the second dose of Covid-19 vaccine (BNT162b2 (Pfizer-BioNTech)), followed by a sudden asthenia, polyarthralgia, throbbing and edema over her lower limbs. She had been given the first dose of the same Covid-19 vaccine 29 days prior to the second without incident. General physical examination was normal. Skin examination showed multiple, erythematous tender, nodules, $10-30 \mathrm{~mm}$ in diameter, over the tibial area. Complete blood count, renal and hepatic tests, antistreptolysin $\mathrm{O}$ titer, antinuclear antibody, thyroid test and chest radiograph and PCR, were carried out, and found to be normal. Histopathology revealed infiltration of deep dermal vessels and subcutaneous fat with lymphomononuclear cells and neutrophils, consistent with erythema nodosum. Treatment with analgesics led to complete resolution of the lesion after three months. The patient has shown no relapse after follow-up for three months.

In conclusion, to our knowledge, this is the first case of EN induced by the second dose of BNT162b2 (PfizerBioNTech) Covid-19 vaccine. It is important for clinicians to be aware of this rare, yet potential, adverse effect to this vaccine.

Keywords: covid-19, vaccination, erythema nodosum, BNT162b2, an mRNA vaccine, side effects.

\section{Introduction}

Erythema nodosum (EN) is a reactive inflammation of the subcutaneous fat characterized clinically by painful tender, nodular, erythematous lesions, typically symmetrically on pretibial surfaces. EN subsides classically in 3 to 6 weeks with mild hyperpigmentation (1). Pathogenesis is thought to be related to deposition of immune complexes in venules of the deep dermis and adipose tissue (2). EN mainly be caused by numerous infective (especially Beta-hemolytic streptococcal infections), autoimmune diseases (especially sarcoidosis) and drugs. Up to 10 percent of erythema nodosum cases are attributed to medicines particularly antibiotics and oral contraceptives (3). EN associated with vaccines has been rarely reported (4). Since March 2020, the Coronavirus disease 2019 (Covid-19) pandemic has affected and caused death of millions of people all over the world and vaccines are considered the most effective strategy to end the pandemic (5). A wide variety of skin reactions occurring after Covid-19 vaccination was described in the literature mainly urticaria, angioedema and maculo-papular eruption (6). EN was once reported to be elicited by viral vector vaccine (ChAdOx1) but never with mRNA ones (7).

We describe herein, a clinical observation of EN induced by BNT162b2, an mRNA vaccine.

Case report

A 75-year-old woman presented with diffuse erythematous painful rounded nodular lesions, located symmetrically over her legs. Six days before, she had received the second dose of Covid-19 vaccine (BNT162b2 (Pfizer-BioNTech)), followed by a sudden asthenia, polyarthralgia, throbbing and edema over her lower limbs. She had a medical history including type-2 diabetes, hypertension and psoriasis and no known drug allergy. She had been given the first dose of the same Covid-19 vaccine 29 days prior to the second without incident. General physical examination was normal. Skin examination showed multiple, erythematous tender, nodules, 10-30 $\mathrm{mm}$ in diameter, over the tibial area (Figure). Complete blood count, renal and hepatic tests, antistreptolysin $\mathrm{O}$ titer, antinuclear antibody, thyroid test and chest radiograph and PCR, were carried out, and found to be normal. Histopathology revealed infiltration of deep dermal vessels and subcutaneous fat with lymphomononuclear cells and neutrophils, consistent with erythema nodosum. Treatment with analgesics (acetaminophen, tramadol...) led to complete resolution of the lesion after three months. The patient has shown no relapse after follow-up for three months. 


\section{Discussion}

We describe a clinical observation of patient who developed erythema nodosum thought to be related to the Covid-19 vaccine. Indeed, a clear temporal relationship was observed between the vaccine administration and the symptoms' onset, the spontaneous remission of the symptomatological pattern and the absence of other attributable aetiologies of the eruption. Based on the Naranjo algorithm, it is probable that the systemic reaction was due to the Covid-19 vaccine (8). EN is regarded as an immune-complex deposition disease affecting venules of the deep dermis and adipose tissue. in contrast to our patient, EN is most commonly observed in young women (between 20 and 50 years) (9). The localization of the EN in the current case was typical since the extensor leg below the knee is the most frequent location, but EN may also occur on other sites such as the upper limbs (2). The majority of EN cases is idiopathic, it may be related to some conditions i.e., tuberculosis, sarcoidosis, and inflammatory bowel disease (2). Drugs are also involved in inducing EN, especially sulphonamides, analgesics, oral contraceptives and proton pump inhibitors (3). Vaccines are uncommon causes of EN as described by some case reports. Involved vaccines were: BacilleCalmette-Guerin Hepatitis B, Human papillomavirus, Malaria, Rabies, Smallpox, Tetanus, diphtheria, and pertussis and Typhoid, and cholera $(4,10,11)$. Interestingly, our case suggests the relationship between the Covid-19 Pfizer vaccine and EN. Cutaneous adverse effects of Covid-19 vaccines have been described recently. Bellinato et al. (12) have summarized the available data related to the cutaneous adverse reactions following Covid-19 vaccines but erythema nodosum induced by Pfizer vaccine is lacking in this review. The most common reported side effects of Covid-19 vaccines were injection site reactions, generally mild or moderate (13). Limited case series or sporadic case reports included exanthemas (14), vascular lesions (15), urticaria (16), eczematous dermatitis (17), autoimmune bullous reactions (18), and severe cutaneous adverse reactions (19). Moreover, the exacerbation of chronic immuno-mediated dermatoses (mainly psoriasis and atopic dermatitis) and reactivations of herpes infection were reported (20,21). Covid-19 vaccine-induced EN was, to our knowledge, described only once previously (7). In that case EN has occurred seven days (six in our patient) after Covid-19 vaccine intake. The time course of development of EN following exposure to a trigger is unpredictable and may be occur between 5 days to 21 months (22). Whereas, the onset of EN could be within shorter duration. For instance, Cohen (4) has reported a case of EN occurring only 48 hours after combined reduced-antigen content tetanus diphtheria, and acellular pertussis. In our patient, we ruled out a Covid-19 infection that might explain the occurrence of EN. In fact, EN was reported to be involved in the clinical picture of Covid-19 infection (23). The vaccine implicated in eliciting EN in the previously reported case was Covishield ${ }^{\mathrm{TM}}$ a Oxford-AstraZeneca Covid-19 vaccine, manufactured in India. Similarly, Rademacher et al., have described two cases of Löfgren's Syndrome (associating bilateral hilar lymphadenopathy, erythema nodosum, and ankle periarthritis) after Covid-19 vaccination by ChAdOx1, Vaxzevria, AstraZeneca (24). Hence, no cases of EN induced by the Pfizer Covid-19 vaccine are reported in the literature yet. In the present case, the EN onset has been after the second dose of Covid-19 vaccine, as it had occurred subsequently to the second in two cases out of three of the published cases. Generally, cutaneous reactions are slightly more common after the first dose compared to the booster (53\% vs.46\%, respectively) (14).

In conclusion, to our knowledge, this is the first case of EN induced by the second dose of BNT162b2 (Pfizer-BioNTech) Covid-19 vaccine. It is important for clinicians to be aware of this rare, yet potential, adverse effect to this vaccine. The literature is likely to reveal more cutaneous reactions induced by Covid-19 vaccination in the future.

1. Requena L, Yus ES. Erythema nodosum. Dermatol Clin. oct 2008;26(4):425-38, v.

2. Schwartz RA, Nervi SJ. Erythema Nodosum: A Sign of Systemic Disease. Am Fam Physician. 1 mars 2007;75(5):695-700.

3. Mert A, Ozaras R, Tabak F, Pekmezci S, Demirkesen C, Ozturk R. Erythema nodosum: an experience of 10 years. Scand J Infect Dis. 2004;36(6-7):424-7.

4. Cohen PR. Combined reduced-antigen content tetanus, diphtheria, and acellular pertussis (tdap) vaccine- 
related erythema nodosum: case report and review of vaccine-associated erythema nodosum. Dermatol Ther. dec 2013;3(2):191-7.

5. Iannone P, Castellini G, Coclite D, Napoletano A, Fauci AJ, Iacorossi L, et al. The need of health policy perspective to protect Healthcare Workers during COVID-19 pandemic. A GRADE rapid review on the N95 respirators effectiveness. PloS One. 2020;15(6):e0234025.

6. Banerji A, Wickner PG, Saff R, Stone CA, Robinson LB, Long AA, et al. mRNA Vaccines to Prevent COVID-19 Disease and Reported Allergic Reactions: Current Evidence and Suggested Approach. J Allergy Clin Immunol Pract. avr 2021;9(4):1423-37.

7. Mehta H, Handa S, Malhotra P, Patial M, Gupta S, Mukherjee A, et al. Erythema nodosum, zoster duplex and pityriasis rosea as possible cutaneous adverse effects of Oxford-AstraZeneca COVID-19 vaccine: report of three cases from India. J Eur Acad Dermatol Venereol JEADV. 21 sept 2021;

8. Naranjo CA, Busto U, Sellers EM, Sandor P, Ruiz I, Roberts EA, et al. A method for estimating the probability of adverse drug reactions. Clin Pharmacol Ther. aout 1981;30(2):239-45.

9. Gilchrist H, Patterson JW. Erythema nodosum and erythema induratum (nodular vasculitis): diagnosis and management. Dermatol Ther. aout 2010;23(4):320-7.

10. Kaliyadan F, Dharmaratnam AM. Erythema nodosum-an association with rabies vaccination. Dermatol Online J. 15 juin 2008;14(6):22.

11. Wu Y-L, Tsai M-H, Liu L-L. Erythema nodosum and hepatitis B: a case report and literature review. J Microbiol Immunol Infect Wei Mian Yu Gan Ran Za Zhi. oct 2008;41(5):437-9.

12. Bellinato F, Maurelli M, Gisondi P, Girolomoni G. Cutaneous Adverse Reactions Associated with SARSCoV-2 Vaccines. J Clin Med. 16 nov 2021;10(22):5344.

13. McMahon DE, Amerson E, Rosenbach M, Lipoff JB, Moustafa D, Tyagi A, et al. Cutaneous reactions reported after Moderna and Pfizer COVID-19 vaccination: A registry-based study of 414 cases. J Am Acad Dermatol. juill 2021;85(1):46-55.

14. Catala A, Munoz-Santos C, Galvan-Casas C, Roncero Riesco M, Revilla Nebreda D, Sola-Truyols A, et al. Cutaneous reactions after SARS-CoV-2 vaccination: a cross-sectional Spanish nationwide study of 405 cases. Br J Dermatol. 13 juill 2021;

15. Larson V, Seidenberg R, Caplan A, Brinster NK, Meehan SA, Kim RH. Clinical and histopathological spectrum of delayed adverse cutaneous reactions following COVID-19 vaccination. J Cutan Pathol. janv 2022;49(1):34-41.

16. Holmes GA, Desai M, Limone B, Love J, Tawfik M, Wong L, et al. A case series of cutaneous COVID-19 vaccine reactions at Loma Linda University Department of Dermatology. JAAD Case Rep. oct 2021;16:53-7.

17. Corbeddu M, Diociaiuti A, Vinci MR, Santoro A, Camisa V, Zaffina S, et al. Transient cutaneous manifestations after administration of Pfizer-BioNTech COVID-19 Vaccine: an Italian single-centre case series. J Eur Acad Dermatol Venereol JEADV. aout 2021;35(8):e483-5.

18. Tomayko MM, Damsky W, Fathy R, McMahon DE, Turner N, Valentin MN, et al. Subepidermal blistering eruptions, including bullous pemphigoid, following COVID-19 vaccination. J Allergy Clin Immunol. sept 2021;148(3):750-1.

19. Annabi E, Dupin N, Sohier P, Garel B, Franck N, Aractingi S, et al. Rare cutaneous adverse effects of COVID-19 vaccines: a case series and review of the literature. J Eur Acad Dermatol Venereol JEADV. dec 2021;35(12):e847-50.

20. Sotiriou E, Tsentemeidou A, Bakirtzi K, Lallas A, Ioannides D, Vakirlis E. Psoriasis exacerbation after COVID-19 vaccination: a report of 14 cases from a single centre. J Eur Acad Dermatol Venereol JEADV. 
dec 2021;35(12):e857-9.

21. Vastarella M, Picone V, Martora F, Fabbrocini G. Herpes zoster after ChAdOx1 nCoV-19 vaccine: a case series. J Eur Acad Dermatol Venereol JEADV. dec 2021;35(12):e845-6.

22. Salvatore MA, Lynch PJ. Erythema nodosum, estrogens, and pregnancy. Arch Dermatol. mai 1980;116(5):557-8.

23. Suter P, Mooser B, Pham Huu Thien HP. Erythema nodosum as a cutaneous manifestation of COVID-19 infection. BMJ Case Rep. 8 juill 2020;13(7):e236613.

24. Rademacher J-G, Tampe B, Korsten P. First Report of Two Cases of Lofgren's Syndrome after SARSCoV-2 Vaccination-Coincidence or Causality? Vaccines. 11 nov 2021;9(11):1313.

\section{Hosted file}

Figures . docx available at https://authorea.com/users/455186/articles/552555-erythema-nodosuminduced-by-covid-19-pfizer-biontech-mrna-vaccine 